brought to the Department of Pediatrics (in KFH- Al Baha or Al Nour Hospital - Makkah) and subjected to: a detailed history, physical \& systemic examinations. Also more urinary, blood and ultrasound studies were done.

Results In Makkah region 1st screening shows that prevalence of hematuria was $0.7 \% \& 0.8 \%$, became by 2 nd screening $0.1 \% \& 0.1 \%$ in male and female, respectively, for proteinuria prevalence by 1 st screening was $1 \% \& 0.8 \%$ became by 2nd screening $0.1 \% \& 0.2 \%$ in male \& female respectively. The prevalence of hypercalciuria by 2 nd screening was $0.1 \%$ $\& 0.1 \%$ in male and female respectively, none of hematuria cases were hypercalciuric.

In Al Baha region prevalence of hematuria by 1 st screening was $0.7 \% \& 0.8 \%$, became by 2 nd screening $0.1 \%$ \& $0.2 \%$ in male \& female, respectively, for proteinuria prevalence by 1 st screening was $0.7 \%$ \& $0.8 \%$ became by 2 nd screening $0.2 \% \& 0.2 \%$ in male and female respectively. The prevalence of hypercalciuria by 2 nd screening $0.2 \% \& 0.2 \%$ in male $\&$ female respectively, and $67.6 \%$ of male hematuria cases, and $71.5 \%$ of female hematuria cases have hypercalciuria.

Conclusion Urinary screening would therefore not only help in early detection but also in the prevention of the deterioration of renal function later in life.

\section{P561 PSEUDOHYPOALDOSTERONISM SECONDARY TO URINARY TRACT INFECTION IN INFANTS}

Sultan Ceren Yıldırım*, Gamze Seval Şahin, Bahriye Atmış, İhsan Turan, Engin Melek, Aysun Karabay Bayazıt. Çukurova University, Adana, Turkey

\subsection{6/archdischild-2019-epa.895}

Hyponatremia with hyperkalemia in infancy is a rare but lifethreatening condition. In the first weeks of life, this scenario is usually associated with aldosterone deficiency due to congenital adrenal hyperplasia (CAH). Pseudohypoaldosteronism (PHA) should be considered in the differential diagnosis of $\mathrm{CAH}$ in infants with hyperkalemia, hyponatremia and metabolic acidosis. Urinary tract infections and/or urinary tract anomalies are the most common causes of type 1 secondary (transient) PHA. Adequate replacement with intravenous saline and antibiotic therapy is necessary to correct electrolyte levels and metabolic acidosis within 24-48 hours. Recognition of type 1 secondary PHA enables appropriate management, thus avoiding unnecessary investigations and treatment. Here, we presented five chidren that emphasized the clinical and biochemical properties of type 1 secondary PHA.

In this study, four male and one female patients whose ages vary between 20 days and 6 months were observed. The mean values of sodium, potassium and bicarbonate were found as $120 \pm 8.9 \mathrm{mmol} / \mathrm{l}, 7.18 \pm 0.98 \mathrm{mmol} / \mathrm{l}, 13.9 \pm 6.19$, respectively for five patients. Renin and aldosterone levels were high in all patients. All patients had urinary tract infection with different urinary tract anomalies. These anomalies were unilateral ureterovesical junction (UVJ) obstruction in two patients, bilateral vesicoureteral reflux (VUR) with posterior urethral valve (PUV) in one patient, PUV with bilateral UVJ obstruction in one patient, and unilateral VUR in one patient. The electrolyte imbalance and metabolic acidosis improved after treatment with intravenous saline and appropriate antibiotic agents. Also, renin and aldosterone levels were decreased to normal limits after the aforementioned treatments.
In this study, our aim was to increase awareness for type 1 secondary PHA among pediatricians. Type 1 secondary PHA should be considered in the presence of hyponatremia, hyperkalemia and metabolic acidosis in infants with urinary tract infections and/or urinary tract anomalies. Also, electrolyte and blood gas monitoring should be done in infants with urinary tract infections.

\section{P562 NOCTURNAL ENURESIS IN CHILDREN: MEDICAL COMORBIDITIES}

${ }^{1}$ Pietro Ferrara*, ${ }^{2}$ Roberta Autuori, ${ }^{2}$ Alessandro Di Lucia, ${ }^{2}$ Flavia Dosa, ${ }^{2}$ Giulia Franceschini, ${ }^{2}$ Diletta Saitta, ${ }^{2}$ Michela Pulcino, ${ }^{2}$ Federica Di Ruscio, ${ }^{2}$ Margherita Zona, ${ }^{2}$ Daniela Grasso, ${ }^{2}$ Rossella Giordano, ${ }^{3}$ Massimo Pettoello-Mantovani. ${ }^{1}$ Institute of Pediatrics, Catholic University Medical School, Rome, Italy; ${ }^{2}$ Campus Bio-Medico University, Rome, Italy; ${ }^{3}$ Department of Pediatrics, Scientific Institute Casa Sollievo della Sofferenza, University of Foggia, Foggia, Italy

\subsection{6/archdischild-2019-epa.896}

Objective To evaluate the characteristics of patients with NE. Design It is a cross-sectional study.

Setting During the physical examination, we sought signs and symptoms of voiding disorders and comorbidities with precise questions posed to the patients and their parents. Further, we assessed family history and behavioral characteristics of patients. Analyses were conducted using the Microsoft Excel 2016 program. We used descriptive statistics to describe patients' information.

Patients We recruited 403 children with Noctunal Enuresis (NE) at the Services of Pediatrics, Campus Bio-Medico University Hospital of Rome between June 2013 and July 2018. We rejected 2 children respectively with a renal agenesis and a chromosomopathy and we enrolled 401 patients, 101 girls (25.2\%) and 300 boys (74.8\%), aged 5-16 years; mean age at first visit $8.8 \pm 2.44$ years. Of the 401 eligible patients, we counted $327(81.5 \%)$ patients with monosymptomatic NE (MNE) and $74(18.5 \%)$ patients with non-monosymptomatic MNE (N-MNE). In the group of MNE children, 242 (74\%) children were boys with mean age at first examination 9.1 years and $85(26 \%)$ were girls with mean age at first examination 7.8 years. In the group of N-MNE children, 58 (78.4\%) children were boys with mean age at first examination 9 years and $16(21.6 \%)$ were girls with mean age at first examination 8.4 years. Among the children with MNE, 322 (98.5\%) had PMNE and 5 (1.5\%) were diagnosed with SMNE.

\section{Interventions Not applicable}

Main outcome measures Signs and symptoms of voiding disorders, comorbidities, family history of NE, behavioral characteristics and school achievement.

Results We noticed that heredity, parasomnias, urogenital abnormalities, constipation and innocence heart murmur are correlated to NE. Notably, 53.6\% (67/125 children) had paternal heredity, $46.4 \%(58 / 125)$ had maternal heredity; $11.2 \%$ $(14 / 125)$ had both paternal and maternal inheritance. 12/401 (3\%) had sleep apnea, 95/401 (23.7\%) had somniloquy and $59 / 401(14.7 \%)$ had bruxism. Children suffered from headaches were 21/401 (5.2\%): $112(27.3 \%)$ had family history of headache. $47 / 300$ boys $(15.7 \%)$ had urogenital-abnormalities: 28/47 (59.6\%) children had balanopreputial adhesions. Cutaneous manifestations of spinal dysraphism such as pilonidal dimple, single and deflected intergluteal cleft or double intergluteal cleft were found in 21/401 (5.2\%) patients. Encopresis 
was found in 12/401 (3\%) children and 58/401 (14.5\%) suffered from constipation. Lastly, a significant percentage of children presented innocent heart murmur (86/401 - 21.4\%). Conclusions Our experience demonstrate that there are many comorbidities that influence the prognosis and the response to the therapy in these children.

\section{P563 PROFILE OF PAEDIATRIC PATIENTS WITH URINARY TRACT INFECTION}

Otilia Frasinariu*, Violeta Streanga, Aniela Rugina, Nicolai Nistor. University of Medicine and Pharmacy Grigore T. Popa, lasi, Romania

10.1136/archdischild-2019-epa.897

Background and aims Urinary tract infection (UTI) is one of the most common pediatric infections. The aim of the study was to evaluate the clinical-epidemiological profile of children with UTI.

Methods The study group consists in 87 children, 55 girls and 32 boys, aged 3 months - 13 years, hospitalised in a Paediatric Clinic during 16 months, diagnosed with urinary tract infections. Diagnostic criteria were the presence of pyuria and/ or bacteriuria on urinalysis and the presence of more than 100.000 colony-forming units (CFU) per $\mathrm{mL}$ of a uropathogen.

Results The distribution of patients by age groups showed a higher prevalence in the age-groups 1-12 months, 24,14\%, and 7-14 years, 29,89, than in children 1-3 years age old, $19,84 \%$ or $3-7$ years age old group, $18,09 \%$, without statistical significance. Depending on the environment, $73,6 \%$ of patients were from urban areas and $22,4 \%$ from rural areas. The incidence of infections was higher in autumn-winter periods, 52\% of cases. Analyzing the way of admission, we found an increased number of the patients sent by the family doctor (63.22\%), followed by patents referred to the hospital in the emergency department, $27.59 \%$ of the patients and $5.75 \%$ of the patients were transferred from another hospital. The etiology of infections was Escherichia coli in $49.43 \%$ of cases, followed by Proteus mirabilis (4.60\%), Enterobacter aerogenes (3.45\%), Staphilococcus epidermidis (3.45\%), Enterococcus faecalis $(5.75 \%)$, Pseudomonas aeruginosa (1.15\%), Streptococcus faecalis $(4.60 \%)$, Candida albicans $(2.30 \%)$. The risk factors were present in $54 \%$ of our patients: $5,74 \%$ presented renal litiasis, 4,6\% duplicated renal collecting system, 10,3\% hidronefrosis, 9,2\% vesico-ureteral reflux, 2 patients neurogenic bladder, 3 patients chronic constipation, 1 patient coalescence of small labia.

Conclusions In urinary tract infection, E. coli is remaining the most prevalence etiological factor in our population: The prevalence is bigger in patients with a malformative risk factor.

\section{\begin{tabular}{|l|l}
\hline P564 A QUALITY IMPROVEMENT PROJECT ON ORAL FOOD \\
\hline
\end{tabular} CHALLENGE}

Amruta Fulmali*, Halima Sadiya Gumi. North Devon District Hospital, Barnstaple, Barnstaple, UK

10.1136/archdischild-2019-epa.898

Introduction This is a Quality Improvement project done in North Devon District Hospital, Barnstaple, UK (level 1 unit) to find out compliance with a Food challenge protocol and to improve our current practice.

We used a food challenge protocol from Royal Devon and Exeter Hospital as a standard protocol.

Methods Total number of food challenges audited were 25 .

All the notes were inspected against following standards: Age, Gender, Type of allergen Challenged, written consent, Time lag between Skin Prick test (SPT) and Food challenge, Risk assessment done or not, allergy focused documentation of clinical examination before and after challenge, Observations before and after challenge, outcome of challenge.

Results Main population was between age 1-5 years with Female to male ratio of 1.08 . We carried out a variety of challenges; majority being baked eggs and milk.

We found out quite a few gaps in our practice.

1. An informed written consent was not taken for any of the challenges.

2. None of the parents were given information leaflet about food challenge.

3. Last skin prick test result was documented in Only $3 / 25$ (12\%) food challenges.

4. Last antihistamine use was documented before 1/25 (4\%) food challenge.

5. None of the children with high risk challenges had IV line done beforehand.

6. $80 \%$ of the time we didn't examine the child after food challenge was complete hence outcome of the challenge was not documented clearly.

7. $44 \%$ of our cohort of population had to wait for $>2$ months after negative SPT disparate recommended waiting time of $2-4$ weeks.

Conclusions Currently we are not following recommended protocol for a food challenge. If a proper protocol is not followed during a food challenge then there are high chances of failed food challenges and anaphylaxis. This might lead to elevated parental anxiety and needless exclusion of food from diet which may lead to essential vitamin/mineral deficiency.

Actions Following this audit, we formed pro-forma which includes all the relevant information required before the food challenge and is easy to follow.

If we gather all the relevant information; then a Junior doctor and nurse will feel comfortable when carrying out a food challenge and outcome of challenge will be accurate and therefore less or no false positives.

Single page pro forma will save time required to clerk these patients on a busy day on the ward.

\section{P565 VALIDATION OF NOVEL GROWTH-PROMOTING AND GROWTH-SUPPRESSING GENES IN NEUROBLASTOMA CELLS}

Dalia Hammouche*, Andrew Stoker. ICH GOSH UCL, London, UK

10.1136/archdischild-2019-epa.899

Background Neuroblastoma is the most common extra-cranial embryonal tumour in children. Treatment outcomes and fiveyear survival rates are very poor, thus for improved results there is a need to understand its genetic and molecular drivers.

Aim This project focused on novel genes that can potentially be targeted to induce growth suppression or increase neural differentiation in Neuroblastoma cells. 